\title{
Subject Status Sponsor Defined Identifier
}

National Cancer Institute

\section{Source}

National Cancer Institute. Subject Status Sponsor Defined Identifier. NCI Thesaurus. Code C117658.

One or more sponsor defined characters used to identify, name, or characterize the subject status. 